\title{
CircRNA hsa_circ_0006220 acts as a tumor suppressor gene by regulating miR-197-5p/CDH19 in triple-negative breast cancer
}

\author{
Yue $\mathrm{Shi}^{1 \wedge}$, Tao $\mathrm{Han}^{2}$, Chong $\mathrm{Liu}^{3}$ \\ ${ }^{1}$ Department of Geriatric Surgery, The First Affiliated Hospital of China Medical University, Shenyang, China; ${ }^{2}$ Department of Oncology, The First \\ Affiliated Hospital of China Medical University, Shenyang, China; ${ }^{3}$ Department of Breast Surgery, The First Affiliated Hospital of China Medical \\ University, Shenyang, China \\ Contributions: (I) Conception and design: Y Shi, C Liu; (II) Administrative support: C Liu; (III) Provision of study materials or patients: C Liu; (IV) \\ Collection and assembly of data: Y Shi, T Han; (V) Data analysis and interpretation: C Liu, Y Shi; (VI) Manuscript writing: All authors; (VII) Final \\ approval of manuscript: All authors. \\ Correspondence to: Chong Liu. Department of Breast Surgery, The First Affiliated Hospital of China Medical University, Shenyang, China. \\ Email: liuchong@cmu.edu.cn; Tao Han. Department of Oncology, The First Affiliated Hospital of China Medical University, Shenyang, China. \\ Email: than1984@sina.com.
}

Background: Triple-negative breast cancer (TNBC) is one of the most aggressive breast cancer (BC) subtypes. Circular ribonucleic acids (circRNAs) are a class of novel stable and conserved forms of ribonucleic acids (RNAs). circRNAs have been documented to be involved in multiple diseases, especially malignancies, through a competing endogenous RNA (ceRNA) mechanism. However, few studies have been conducted on the function of circRNAs in TNBC. Previously, hsa_circ_0006220 was found to be downregulated in BC tissues. The present study sought to explore the mechanism of hsa_circ_0006220 in TNBC progression.

Methods: A real-time polymerase chain reaction (PCR) was used to validate the expression of hsa circ_0006220 in TNBC tissues and cells. In addition, 3-(4,5-dimethylthiazol-2-yl)-2,5-diphenyl-2Htetrazolium bromide (MTT), wound-healing and Transwell assays were conducted to measure the inhibition effects of hsa_circ_0006220 on TNBC cells in vitro. Further, a dual-luciferase reporter assay was performed to confirm the interaction between hsa_circ_0006220 and miR-197-5p. A mimic and an inhibitor of miR197-5p were constructed to confirm the downstream mechanism of hsa_circ_0006220 in TNBC cells.

Results: Hsa_circ_0006220 was more downregulated in TNBC than other subtypes of BC tissues and cell lines. In vitro data showed that hsa_circ_0006220 remarkably inhibited the proliferation, migration, and invasion of TNBC cells. Further, hsa_circ_0006220 was confirmed to be a sponge of miR-197-5p, and to indirectly regulate CDH19 expression. A rescue assay indicated the biological function of the hsa circ_0006220/miR-197-5p/CDH19 pathway in TNBC cells.

Conclusions: hsa_circ_0006220 plays an inhibitory role in TNBC progression. It might be a potential diagnostic marker and therapeutic target for TNBC patients.

Keywords: Triple-negative breast cancer (TNBC); circular RNA; hsa_circ_0006220; mechanism

Submitted May 19, 2021. Accepted for publication Jul 13, 2021.

doi: 10.21037/atm-21-2934

View this article at: https://dx.doi.org/10.21037/atm-21-2934

\footnotetext{
$\wedge$ ORCID: 0000-0002-3352-9045.
} 


\section{Introduction}

Breast cancer (BC) is one of the most common malignant tumors among women (1). Triple-negative breast cancer (TNBC) is one of the most aggressive BC subtypes, which is characterized by an absence of estrogen receptor (ER), progesterone receptor (PR), and human epidermal growth factor receptor 2 (HER-2) (2). Compared to other subtypes of $\mathrm{BC}$, the treatment of TNBC is limited and the prognosis is poor due to a lack of therapeutic targets (3). Thus, novel prognostic markers and molecular therapeutic targets for TNBC patients need to be discovered urgently.

Circular ribonucleic acids (circRNAs) are novel endogenous ribonucleic acid (RNA) molecules (4). Compared to linear RNAs, circRNAs are covalently closed continuous loops and could resist digestion by RNAse $\mathrm{R}$ (5-7). Recently, circRNAs have been viewed as pivotal regulators of a wide range of biological processes, and notably, act as a micro RNA (miRNA) sponge in many tumors $(8,9)$. $\mathrm{Li}(10)$ reported that silencing circDDX17 promoted cell proliferation, migration, and invasion, and inhibited apoptosis in colorectal cancer. Circ-FOXM1 promotes cell progression by sponging miR-1304-5p in non-small cell lung cancer (11). In TNBC, circKIF4A was reported to be a prognostic factor and regulate cell proliferation and migration by sponging miR-375 (12). It has been suggested that the circEPSTI1-miR-4753/6809BCL11A axis affects the proliferation and apoptosis of TNBC (13). However, the roles of circRNAs in TNBC remain unclear; thus, further research needs to be conducted to elucidate the functions and mechanisms of circRNAs.

In our previous study, hsa_circ_0006220 was found to downregulate $\mathrm{BC}$ cells and tissues by a circRNA microarray and real-time polymerase chain reaction (RT-PCR). Further, 5 potential target miRNAs were predicted, and hsa_circ_0006220 was confirmed to modulate miR-197-5p in BC cells (14). In addition, Shaker et al. reported that the level of miR-197 was notably increased in the serum of BC patients (15). In the present study, hsa_circ_0006220 was found to be more lowly expressed in TNBC cell lines than other BC cell lines by RT-PCR. Thus, we speculated that hsa circ_0006220 might affect TNBC progression by regulating miR-197-5p. Consequently, the possible relationship between hsa_circ_0006220 and the development of TNBC cells was analyzed in vitro. The relationship between hsa_circ_0006220 and miR-197-5p was also examined. In brief, this study showed that hsa_circ_0006220 may act as a tumor suppressor gene and thus could serve as a potential therapeutic target for
TNBC. We present the following article in accordance with the MDAR reporting checklist (available at https://dx.doi. org/10.21037/atm-21-2934).

\section{Methods}

\section{Sample collection}

A total of 57 BC patients (aged from 27 to 81 years old) participated in this study. All patients were primary female BC patients who had been treated at The First Affiliated Hospital of China Medical University between January 2010 and December 2015. The patients did not receive chemotherapy or radiotherapy before surgery. Tissue specimens, including 11 TNBC tissues and 46 other BC tissue subtypes, were obtained after surgical resection and stored in an ultra-low temperature freezer (Haier, China). The study was approved by the Medical Ethics Committee of China Medical University (No. AF-0G-03-1.0-02). All of the procedures performed in this study involving human participants were conducted in accordance with the Declaration of Helsinki (as revised in 2013). Informed consent was obtained from all of the patients.

\section{Cell cultures}

The human BC cell lines (i.e., BT-549, MDA-MB-231, MDA-MB-468, SK-BR-3, T47D, and MCF-7) and a normal mammary epithelial cell line (i.e., MCF-10A) used in this study were purchased from the Cell Bank of Chinese Academy of Sciences. Human Embryonic Kidney 293T (HEK 293T) cells were preserved at our laboratory. The MDA-MB-231 cells were cultured in Dulbecco's Modified Eagle Medium (Gibco, Carlsbad, CA, USA), containing 10\% fetal bovine serum (FBS; Hyclone, Logan, USA). The T47D cells were cultured in Roswell Park Memorial Institute Medium 1640 (Gibco, Carlsbad, CA, USA), containing 10\% FBS. Michigan Cancer Foundation-10A (MCF-10A) cells were cultured in Mammary Epithelial Basal Medium (iCell Bioscience, Shanghai, China). The other cell lines were cultured appropriately. All the cell lines were free of mycoplasma infection and were maintained at $37^{\circ} \mathrm{C}$ with $5 \%$ carbon dioxide $\left(\mathrm{CO}_{2}\right)$ in a humidified incubator.

\section{RT-PCR}

Total RNA was extracted from the tissues or cells using a simple total RNA kit (BioTeke, Beijing, China). The 
purity and concentration of the RNAs were examined using a NANO 2000 spectrophotometer (Thermo Scientific, Waltham, MA, USA), and then reverse transcribed into complementary deoxyribonucleic acid (cDNA) by Moloney Murine Leukemia Virus Reverse Transcriptase (BioTeke). An Exicycler ${ }^{\mathrm{TM}} 96$ RT-PCR machine (BIONEER, Daejeon, Korea) was used to perform the RT-PCR analysis and calculate the levels of cDNAs using the SYBR-Green method (Takara Bio, Dalian, China). $\beta$-actin was used as an endogenous control for the normalization of circRNA and messenger RNA (mRNA), while U6 was used for the miRNA. The relative expressions of RNAs were analyzed using the $2^{-\triangle \triangle C T}$ method. Table S1 provides details of the primers of genes (Sangon Biotech, Shanghai, China).

\section{Vector construction and cell transfection}

To overexpress hsa_circ_0006220, the mature sequence of hsa_circ_0006220(chr17, 35800605-35800763) was synthesized and cloned into a vector (Genscript, Nanjing, China), named "ov-circ." A mock vector with no hsa_ circ_0006220 sequence served as the control, and was named “ov-NC." To knock down hsa_circ_0006220, small inhibitory RNA (siRNA) targeting the back-splice junction site of hsa_circ_0006220 or a negative control were synthesized and constructed into plasmid vectors (Genscript, Nanjing, China) that were named "sh-circ" and "sh-NC," respectively. miR-197-5p mimics and inhibitors were built by GenePharma (Shanghai, China). The vectors, mimics, and inhibitors were transiently transfected into BC cells with Lipofectamine 2000 (Invitrogen, Carlsbad, CA, USA) in accordance with the manufacturer's protocols. The sequences of hsa_circ_0006220 siRNA and negative control (si-NC) are set out in Table S2. The miR-197-5p inhibitor and negative control sequences are set out in Table S3.

\section{MTT assay}

A density of $3 \times 10^{3}$ cells were seeded in each well of 96-well plates. $5 \mathrm{mg} / \mathrm{mL}$ 3-(4,5-dimethylthiazol-2-yl)-2, 5-diphenyl-tetrazolium bromide (MTT) (Sigma Aldrich, St. Louis, Missouri) was added to each well at $0,24,48$, and 72 hours. They were then incubated at $37^{\circ} \mathrm{C}$ for $4 \mathrm{~h}$. Next, the medium was removed and $200 \mu \mathrm{L}$ of dimethyl sulfoxide (DMSO) was added to homogenize the formazan crystals. The optical density at $570 \mathrm{~nm}$ of each well was detected using an automatic microplate reader (BioTek, Vermont, USA).

\section{Wound-bealing assay}

The $6 \times 10^{5}$ post-transfected cells were seeded in 6-well plates and incubated for $48 \mathrm{~h}$. They were then treated with a serum-free culture containing $1 \mu \mathrm{g} / \mathrm{mL}$ of Mitomycin $\mathrm{C}$ (Sigma Aldrich) for $1 \mathrm{~h}$. Next, the wounds were produced in the middle of each well using $200 \mu \mathrm{L}$ pipette tips. The cells were washed and incubated with a serum-free culture, and the wound closing was photographed by a phase contrast microscope (Motic, Xiamen, China). After being incubated at $37{ }^{\circ} \mathrm{C}$ with $5 \% \mathrm{CO}_{2}$ for $24 \mathrm{~h}$, the widths of the wounds were captured and the migration rates were calculated.

\section{Transwell assay}

For the migration assays, the cells were seeded in the upper chambers (Corning Incorporated, Corning, NY, USA) at a density of $2 \times 10^{4}$ and cultured with $200 \mu \mathrm{L}$ of serum-free medium. The lower chambers were filled with $800 \mu \mathrm{L}$ of medium containing $20 \%$ FBS. After being incubated at $37{ }^{\circ} \mathrm{C}$ with $5 \% \mathrm{CO}_{2}$ for $24 \mathrm{~h}$, the cells that migrated to the bottom of the filter were fixed with $4 \%$ paraformaldehyde for $20 \mathrm{~min}$ and stained with $0.5 \%$ crystal violet dyes (Amresco, USA), and then photographed and counted under an inverted phase contrast microscope (Motic).

For the invasion assays, $2 \times 10^{4}$ cells were seeded into $200 \mu \mathrm{L}$ of serum-free medium in upper chambers coated with Matrigel (BD Biosciences, NJ, USA). An $800-\mu \mathrm{L}$ medium containing $20 \%$ FBS was added to the lower chambers. Briefly, after $24 \mathrm{~h}$, the cells in the lower chambers were stained and photographed by an inverted phase contrast microscope (Motic).

\section{Dual-luciferase reporter assay}

The sequences of wild-type and mutated hsa_circ_0006220 without miR-197-5p binding sites were synthesized and subcloned into luciferase reporter vectors pmirGLO (Genscript), named "circ-WT," and "circ-Mut," respectively. These vectors and the miR-197-5p mimics or negative controls were co-transfected into HEK 293T cells using the Lipofectamine 2000 transfection reagent according to the manufacturer's instructions. After $48 \mathrm{~h}$ of incubation, the relative luciferase activity was measured by a Dual-Luciferase Assay Kit (KeyGEN BioTECH). The luciferase activity was measured normalized to the Renilla luciferase internal control using the Tecan infinite M200 Pro luminometer (Tecan, Männedorf, Switzerland). 


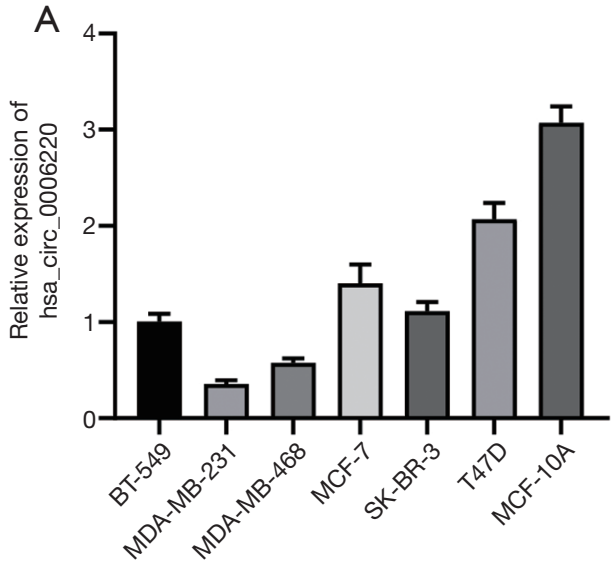

B

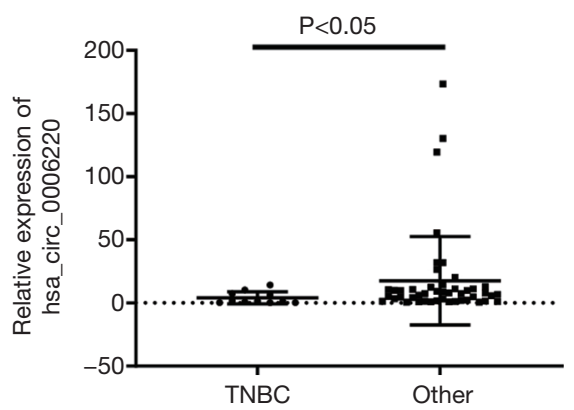

Figure 1 hsa_circ_0006220 was downregulated in TNBC tissues and cell lines. (A) The expression of hsa_circ_0006220 in TNBC cell lines (BT-549, MDA-MB-231, and MDA-MB-468), other subtypes of BC cell lines (MCF-7, SK-BR-3, and T47D), and MCF-10A by RT-PCR. (B) Comparing the levels of hsa_circ_0006220 between TNBC ( $\mathrm{n}=11)$ and other subtypes of BC tissues (n=46) using RT-PCR. Values are presented as mean \pm standard deviation. TNBC, triple-negative breast cancer.

\section{Western blotting}

The proteins in the tissues and cells were extracted using a radio immunoprecipitation assay (RIPA) lysis buffer (Beyotime, Haimen, China) with $1 \mathrm{mM}$ of phenylmethylsulfonyl fluoride (Beyotime), and quantified by a bicinchoninic acid kit (Beyotime). A total of $40 \mu \mathrm{g}$ $(20 \mu \mathrm{L})$ proteins from each sample were separated with sodium dodecyl sulfate polyacrylamide gel electrophoresis, and transferred to polyvinylidene difluoride membranes (Millipore, Bedford, MA, USA). The membranes were incubated at room temperature for $1 \mathrm{~h}$ with $5 \%$ milk, and then incubated with the appropriate primary antibody overnight at $4{ }^{\circ} \mathrm{C}$. A specific primary antibody, antiCDH19 (1:400), was purchased from Proteintech (Wuhan, China), and $\beta$-actin (1:1,000, Wanleibio, Shenyang, China) served as the internal control. Consequently, horseradish peroxidase-conjugated secondary antibody $(1: 5,000$, Wanleibio) and electrochemiluminescence western blotting detection reagents (Wanleibio) were used to visualize the target proteins. The images were finally collected by an enhanced chemiluminescence system (Beyotime).

\section{Statistical analysis}

A statistical analysis was performed using GraphPad Prism 7.0 software (GraphPad Software, CA, USA). The $t$-test with a Wilcoxon signed-rank test was used to compare the differences between the two groups. A one-way analysis of variance with a Kruskal-Wallis test was used for the analyses among the multiple groups. Pearson correlation coefficients were used for the correlation analyses. The results are expressed as mean \pm standard deviation $(\mathrm{SD})$. A P value less than 0.05 was viewed as statistically significant. All experiments were repeated thrice.

\section{Results}

\section{Downregulation of bsa_circ_0006220 in TNBC tissues and cells}

In our previous study, hsa_circ_0006220 was identified to be downregulated in $\mathrm{BC}$ tissues relative to the paired noncancerous tissues using a circRNA microarray and RTPCR (14). In the present study, hsa_circ_0006220 was shown to be more obviously downregulated in BC cells than normal breast epithelial cells, MCF-10A (see Figure 1A) by RT-PCR. Additionally, we found that its levels in TNBC cells (BT-549, MDA-MB-231, and MDA-MB-468) were much lower than those of other subtypes of $\mathrm{BC}$ cells (i.e., MCF-7, SK-BR-3, and T47D; see Figure 1A). Based on these results, we speculated that hsa_circ_0006220 might affect TNBC progression. Subsequently, the levels of hsa circ_0006220 were examined in 11 TNBC tissues and 46 other BC tissue subtypes by RT-PCR. The results showed that it was notably downregulated in TNBC tissues $(\mathrm{P}<0.05$; see Figure $1 B$ ). Thus, we chose MDA-MB-231 (which had the lowest level of hsa_circ_0006220 among TNBC cells) 
and T47D (which had the highest level of hsa_circ_0006220 among other subtypes of BC cells) for our next functional investigation of hsa_circ_0006220.

\section{bsa_circ_0006220 inbibits proliferation, migration, and invasion in TNBC cells}

An overexpression vector of hsa_circ_0006220 and a siRNA plasmid vector were also constructed (see Figure 2A). We overexpressed hsa_circ_0006220 in MDA-MB-231 cells and downregulated it in T47D cells in our next functional investigations, and examined the level of hsa_circ_0006220 by RT-PCR (see Figure 2B). Notably, the expression of the linear transcript TADA2A was not affected when hsa_circ_0006220 was overexpressed or knocked down in BC cells (see Figure 2C). Next, MTT assays were carried out to test the effects of hsa_circ_0006220 on cell proliferation. The results suggested that the cell viability of MDA-MB-231 cells was inhibited significantly after the upregulation of hsa_circ_0006220 at $72 \mathrm{~h}(\mathrm{P}<0.0001)$. Conversely, the viability of $\mathrm{T} 47 \mathrm{D}$ cells increased significantly after the downregulation of hsa_circ_0006220 $(\mathrm{P}<0.0001$; see Figure 2D). Next, wound-healing assays and Transwell assays were performed on BC cells to verify the effects of hsa_circ_0006220 on cell migration and invasion. The cell migration rate of MDA-MB-231 cells was markedly suppressed by the upregulation of hsa_circ_0006220 $(\mathrm{P}<0.0001)$, and was notably enhanced in T47D cells by the downregulation of hsa_circ_0006220 $(\mathrm{P}<0.05$; see Figure 2E). Similarly, the Transwell assays showed that the upregulation of hsa_circ_0006220 significantly inhibited the cell migration $(\mathrm{P}<0.0001$; see Figure $2 F)$ and cell invasion $(\mathrm{P}<0.0001$; Figure $2 G)$ of MDA-MB-231 cells, while the downregulation of hsa_circ_0006220 produced the opposite results in T47D cells (see Figure 2F,2G). These results showed that the proliferation, migration, and invasion of TNBC cells were inhibited by hsa_circ_0006220 in vitro.

\section{bsa_circ_0006220 acts as a sponge of miR-197-5p}

In our previous study, we identified the 5 miRNAs most likely to be sponged by hsa_circ_0006220, and examined their level changes after the upregulation of hsa_circ_0006220 in MDA-MB-231 (14). Among the 5 miRNAs, miR-197-5p was significantly decreased when overexpressing hsa_circ_0006220; thus, it might be a target of hsa_circ_0006220. In addition, it has been reported that miR-197 is upregulated in male BC (15), and
TNBC (16). In this study, the regulation of miR-197-5p by hsa_circ_0006220 was verified in MDA-MB-231 and T47D cells (see Figure 3A). Further, miR-197-5p was found to be markedly more elevated in 11 TNBC tissues than 46 other subtypes of BC tissues by RT-PCR $(\mathrm{P}<0.05$; see Figure $3 B)$. Additionally, a Pearson correlation analysis of all $57 \mathrm{BC}$ tissue samples revealed a significantly negative correlation between the level of miR-197-5p and hsa_circ_0006220 $(\mathrm{r}=-0.2689, \mathrm{P}=0.0431$; see Figure $3 C)$. Unlike hsa circ_0006220, miR-197-5p was more obviously elevated in BC cell lines than MCF-10A using RT-PCR, and was much higher in TNBC cell lines (see Figure 3D). Thus, a dualluciferase reporter assay was performed in HEK $293 \mathrm{~T}$ cells to examine whether hsa_circ_0006220 served as a sponge for miR-197-5p. The results showed that transfections with the miR-197-5p mimics significantly reduced the luciferase activity with hsa_circ_0006220 wild-type compared to that of miR-197-5p NC $(\mathrm{P}<0.001$; see Figure $3 E)$. Conversely, the miR-197-5p mimics did not decrease the luciferase activity in the two circ-Mut groups (see Figure 3E). Thus, hsa_circ_0006220 might serve as a sponge for miR-197-5p through the predicted binding site.

\section{CDH19 is a target of miR-197-5p and is regulated by bsa circ_0006220}

Next, to examine the role of hsa_circ_0006220 in TNBC cells, the downstream target of miR-197-5p was predicted by miRanda. We found that CDH19 was likely to have the same miRNA response element (MRE) of miR-197-5p (see Figure $4 A$ ). In addition, CDH19 was appeared to represent a novel cadherin and was involved in establishing and maintaining intercellular connections (17-19). Thus, we speculated that CDH19 might act as a tumor suppressor gene. Consequently, in the present study, CDH19 was verified to downregulate in TNBC tissues by RT-PCR $(\mathrm{P}<0.05$; Figure $4 B)$. The Pearson correlation analysis showed that CDH19 was positively associated with hsa_circ_0006220 expression in $57 \mathrm{BC}$ tissues $(\mathrm{r}=0.8731, \mathrm{P}<0.0001$; see Figure $4 C$ ). Further, the level of CDH19 was also more downregulated in $\mathrm{BC}$ cell lines than MCF-10A (see Figure 4D). We then examined whether hsa_circ_0006220 affects the expression of CDH19 to explore the role of the hsa_circ_0006220/miR-197-5p/CDH19 pathway in TNBC cells. An overexpression of hsa_circ_0006220 notably increased the level of CDH19 in MDA-MB-231 cells $(\mathrm{P}<0.0001)$, while the downregulation of hsa_circ_0006220 
A
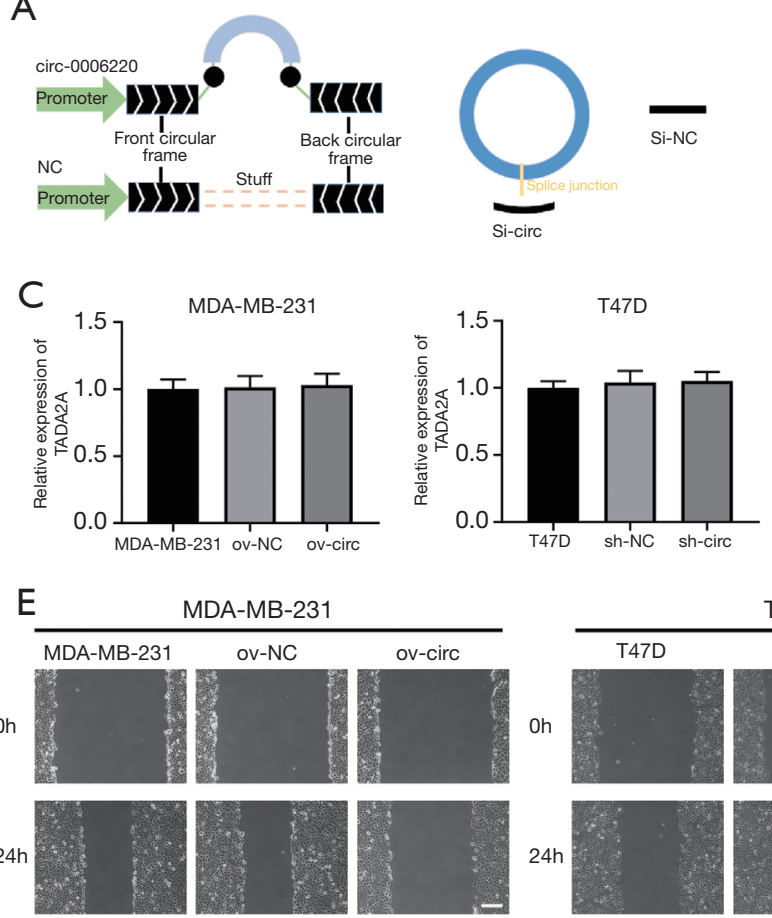

B

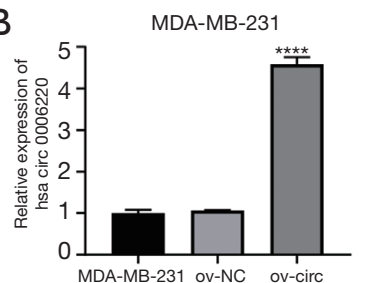

D
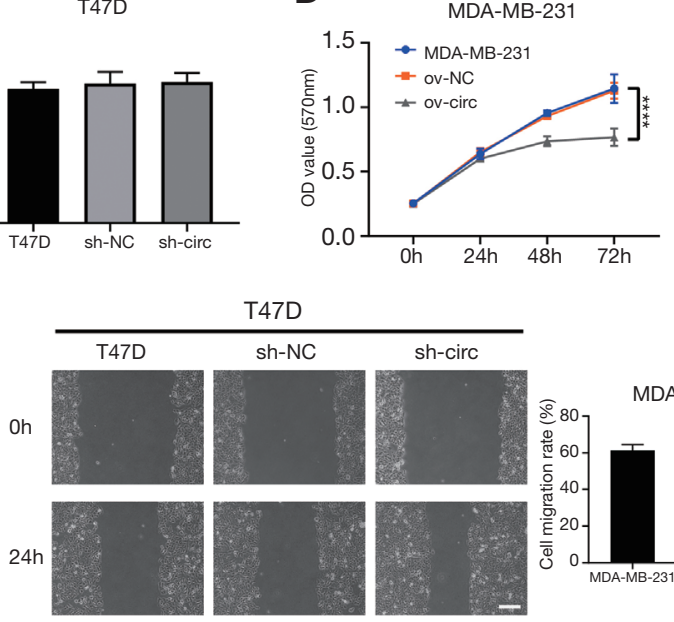

T47D
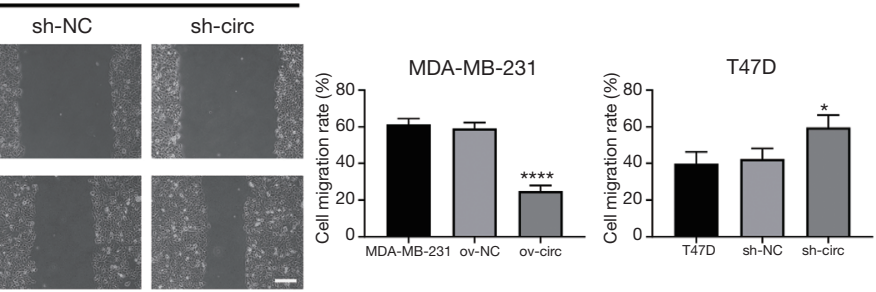

F
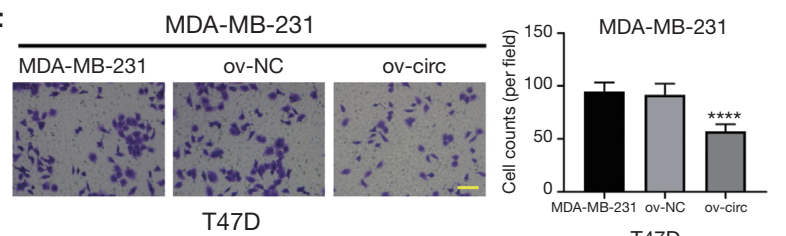

G

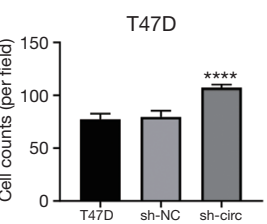

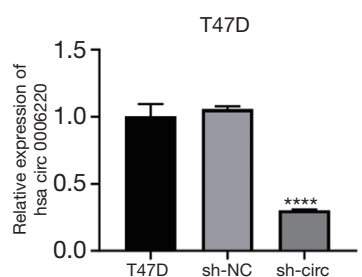

T47D

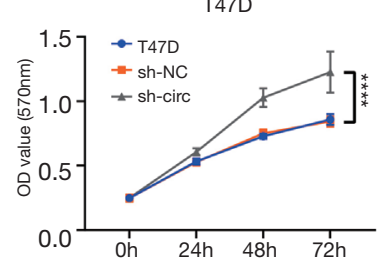

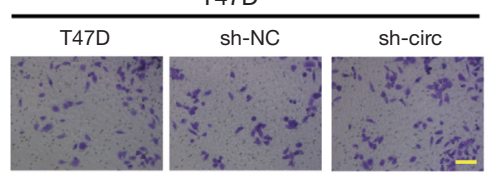
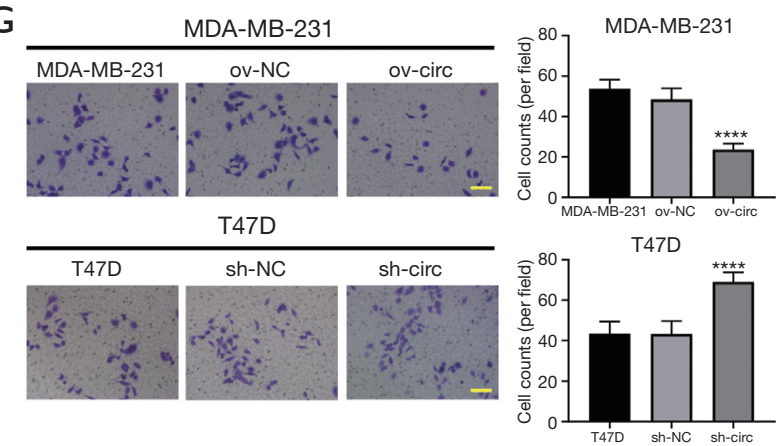

Figure 2 hsa_circ_0006220 inhibited TNBC cell growth, migration, and invasion. (A) Schematic illustration of the vector and siRNAs of hsa_circ_0006220. (B,C) 24 hours after transfecting MDA-MB-231 with the hsa_circ_0006220 expression vector (ov-circ) or mock (ov-NC), and T47D cells with the siRNA (sh-circ) or negative control (sh-NC), the relative expressions of hsa_circ_0006220 and TADA2A were verified by RT-PCR. (D) The growth curves of cells transfected with the specified vectors were analyzed by MTT assays. (E) Cell migration capacities were examined by wound-healing assays after being transfected with the specified vectors. Scale bar, 200 pm. (F,G) Cell migration and invasion abilities were analyzed by Transwell assays after being transfected with the specified vectors. Stain method: $0.5 \%$ crystal violet dyes. Scale bar, $100 \mu \mathrm{m}$. Data are presented as mean \pm standard deviation $(\mathrm{n}=3)$. *, $\mathrm{P}<0.05 ;{ }^{* * * *}, \mathrm{P}<0.0001$.

decreased the level of CDH19 in T47D $(\mathrm{P}<0.0001$; see Figure $4 E$ ). Similarly, the western blot showed that the protein level of CDH19 was also positively regulated (up or down) by the regulation of hsa_circ_0006220 in the 2 BC cell lines (see Figure 4F,4G). All these data suggested that hsa_circ_0006220 might affect the level of CDH19 by sponging miR-197-5p in TNBC.

\section{bsa_circ_0006220 inhibits TNBC cell progression through the bsa_circ_0006220/miR-197-5p/CDH19 patbway}

To confirm that hsa_circ_0006220 functions in TNBC cells via the hsa_circ_0006220/miR-197-5p/CDH19 pathway, miR-197-5p mimics and inhibitors were constructed and co-transfected into MDA-MB-231 and T47D cells. 

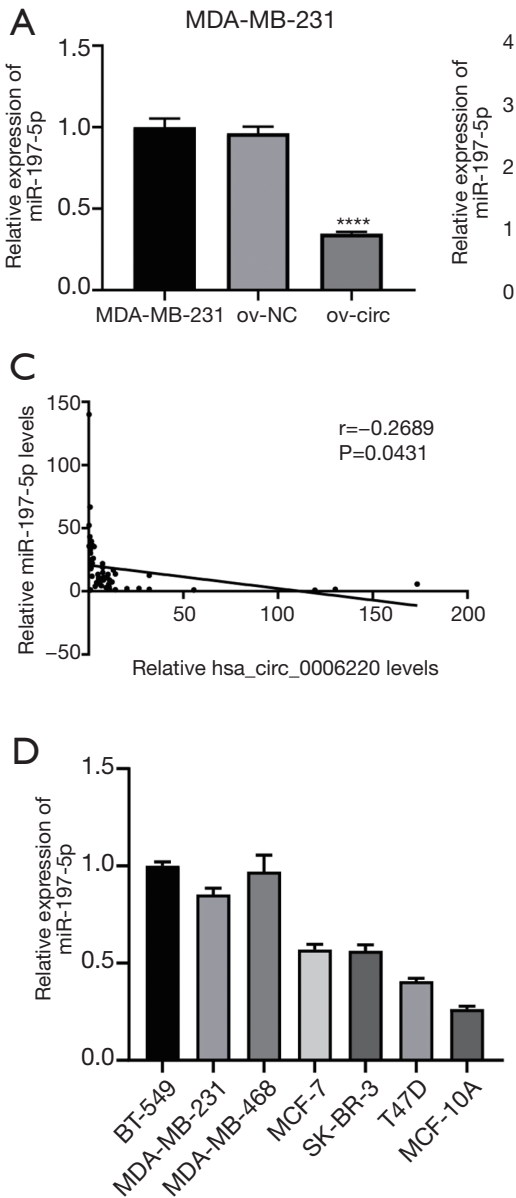
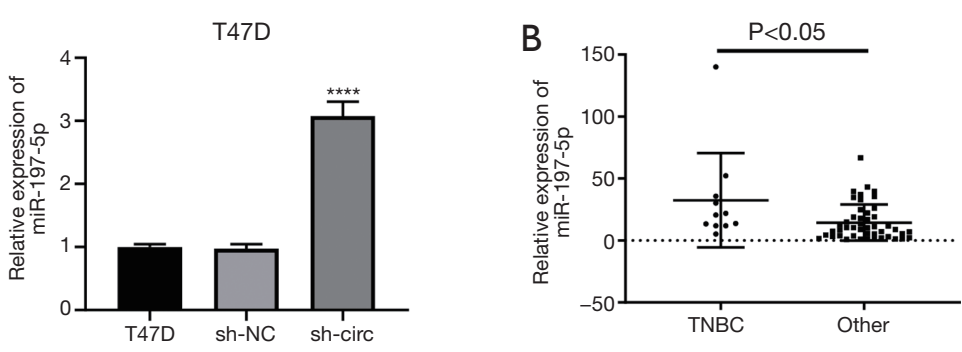

$\mathrm{E}$
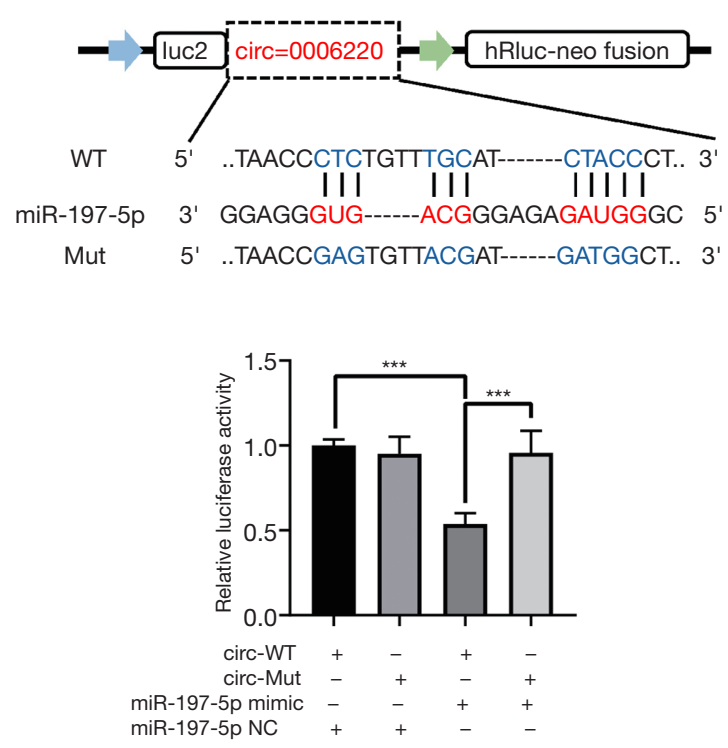

Figure 3 hsa_circ_0006220 regulates and functions as a sponge of miR-197-5p. (A) RT-PCR analysis of miR-197-5p expression in MDA-MB-231 cells transfected with the hsa_circ_0006220 expression vector (ov-circ) or the mock (ov-NC), and T47D cells with hsa_circ_0006220 siRNA (sh-circ) or the negative control (sh-NC). (B) Examination of the relative expression of miR-197-5p in TNBC tissues ( $\mathrm{n}=11$ ) and other subtypes of BC tissues ( $\mathrm{n}=46$ ) by RT-PCR. (C) Pearson correlation analysis of miR-197-5p and hsa_circ_0006220 expression in all BC tissues ( $\mathrm{n}=57$ ). (D) The relative expression of miR-197-5p in cell lines was analyzed by RT-PCR. (E) Schematic illustration of the hsa_circ_0006220 WT and the hsa_circ_0006220 mutant (Mut) luciferase reporter vectors on the predicted binding sites of miR-197-5p. After transfection with the hsa_circ_0006220 wild-type (circ-WT) or the hsa_circ_0006220 mutant (circ-Mut) and the miR-197-5p mimic or the miR-197-5p NC in HEK 293T cells, relative luciferase activities were detected in each group. Data are expressed as mean \pm standard deviation $(\mathrm{n}=3) .{ }^{* * *}, \mathrm{P}<0.001$; ${ }^{* * *}, \mathrm{P}<0.0001$. WT, wild-type; NC, negative control; TNBC, triple-negative breast cancer.

The expression of hsa_circ_0006220 was then examined in MDA-MB-231 and T47D cells by RT-PCR. We found that the miR-197-5p mimics reduced the level of hsa_circ_0006220, while the miR-197-5p inhibitors had the opposite effect (see Figure $5 A$ ). In the MTT assays, the overexpression of hsa_circ_0006220 dramatically reduced the cell viability of MDA-MB-231 cells; however, this effect was reversed by the miR-197-5p mimics. Additionally, the downregulation of hsa_circ_0006220 increased the cell viability of T47D, but was counteracted by the miR-197-5p inhibitors (see Figure 5B). Further, the wound-healing assays showed that the miR-197-5p mimics abolished the reduction of the cell migration rate of MDA-MB-231 cells by the overexpression of hsa_circ_0006220, while the miR$197-5 p$ inhibitors reversed the elevation of the cell migration rate of T47D cells by silencing hsa_circ_0006220 (see Figure 5C). Briefly, the Transwell assay produced similar results, which showed that the effects on cell migration and invasion by hsa_circ_0006220 could be rescued by the miR-197-5p mimics or inhibitors, respectively (see Figure 5D,5E). 

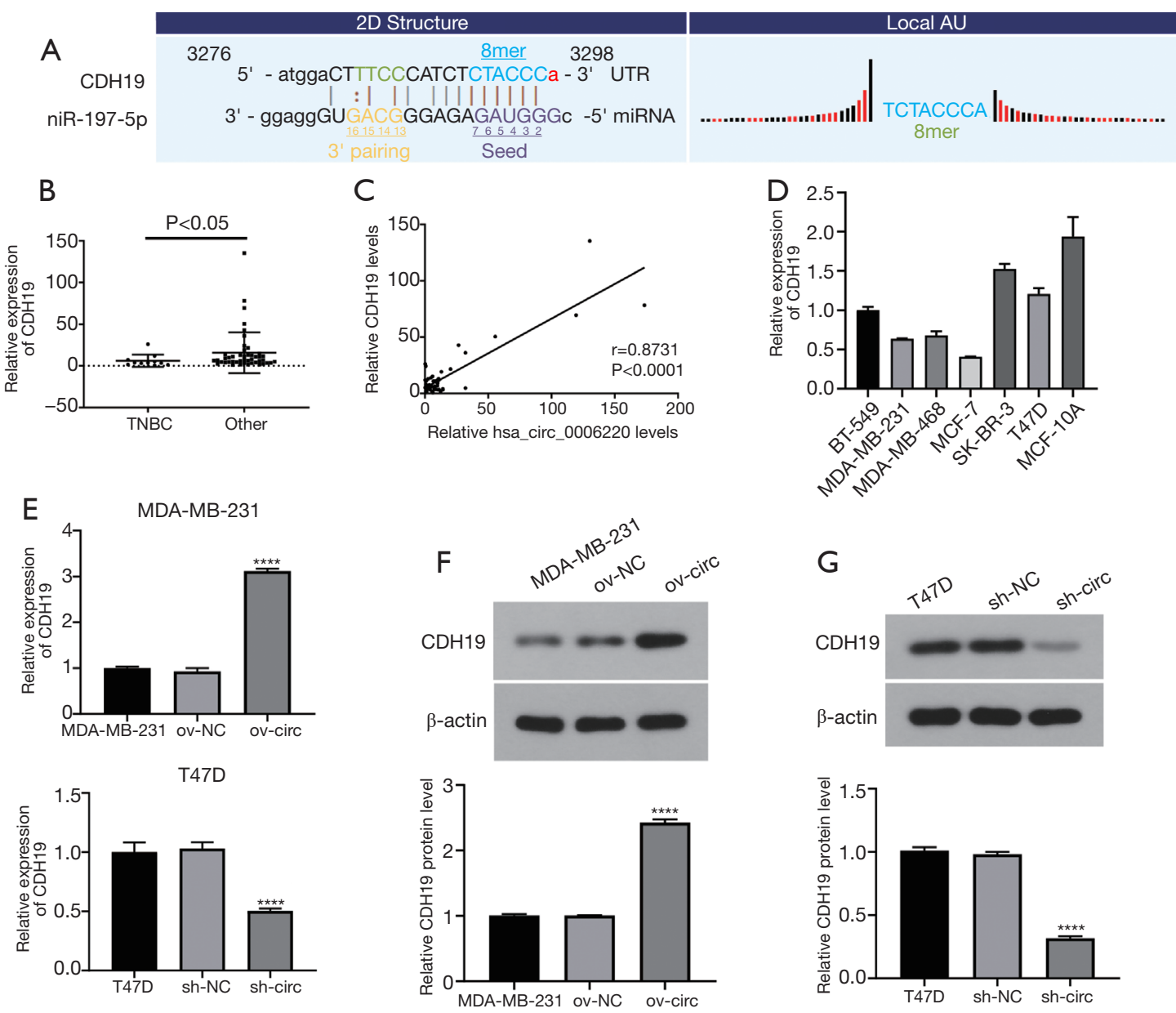

Figure 4 CDH19 was regarded as a target gene of miR-197-5p and regulated by hsa_circ_0006220. (A) Prediction of miR-197-5p binding sites on CDH19 using miRanda. The binding sites are presented as solid lines. (B) Validation of the relative expression of CDH19 in TNBC tissues ( $\mathrm{n}=11$ ) and other subtypes of BC tissues (n=46) by RT-PCR. (C) Pearson correlation analysis of CDH19 and hsa_circ_0006220 expression in all BC tissues ( $\mathrm{n}=57$ ). (D) Relative mRNA levels of CDH19 in cell lines was detected by RT-PCR. (E) RT-PCR analysis of CDH19 mRNA levels in MDA-MB-231 cells transfected with the hsa_circ_0006220 expression vector (ov-circ) or a mock (ov-NC), and in T47D cells with the hsa_circ_0006220 siRNA (sh-circ) or a negative control (sh-NC). (F,G) Relative protein levels of CDH19 were examined in cells transfected with the specified vectors using western blot. Data are expressed as mean \pm standard deviation $\left(\mathrm{n}=3\right.$ ). ${ }^{* * *}$, $\mathrm{P}<0.0001$. TNBC, triple-negative breast cancer.

\section{bsa_circ_0006220 regulates CDH19 expression through the hsa_circ_0006220/miR-197-5p/CDH19 pathway}

Next, the expression of miR-197-5p was verified when co-transfected with miR-197-5p mimics or inhibitors in MDA-MB-231 or T47D cells (see Figure 6A). The results of the RT-PCR and western blot analyses suggested that the upregulation of hsa_circ_0006220 elevated the mRNA and protein levels of CDH19 in MDA-MB-231 cells, while the knockdown of hsa_circ_0006220 reduced the levels of CDH19 in T47D cells. The miR-197-5p mimics or inhibitors abolished each aforementioned effect, respectively (see Figure 6B,6C). These results showed that CDH19 appeared to be regulated by hsa_circ_00062220 though the hsa_circ_0006220/miR-197-5p/CDH19 pathway, and thus affected the development of TNBC cells. 

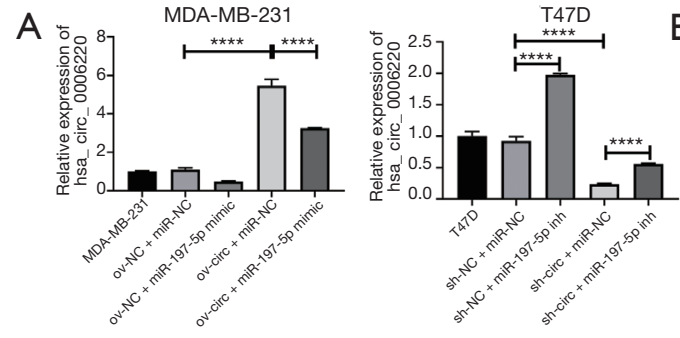

C
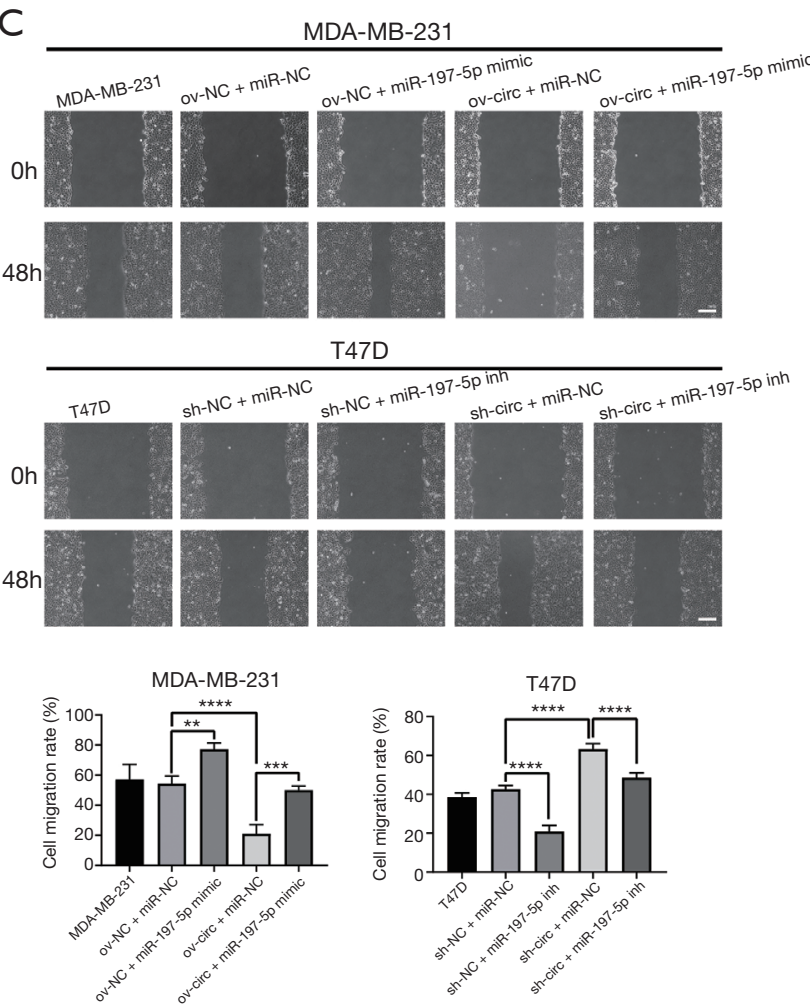

B
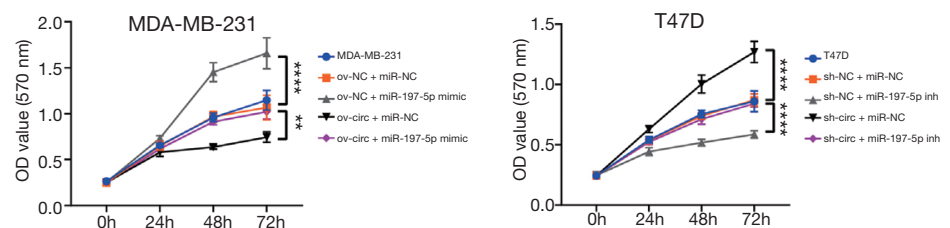

D

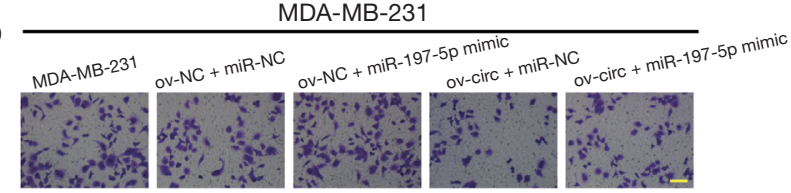

E
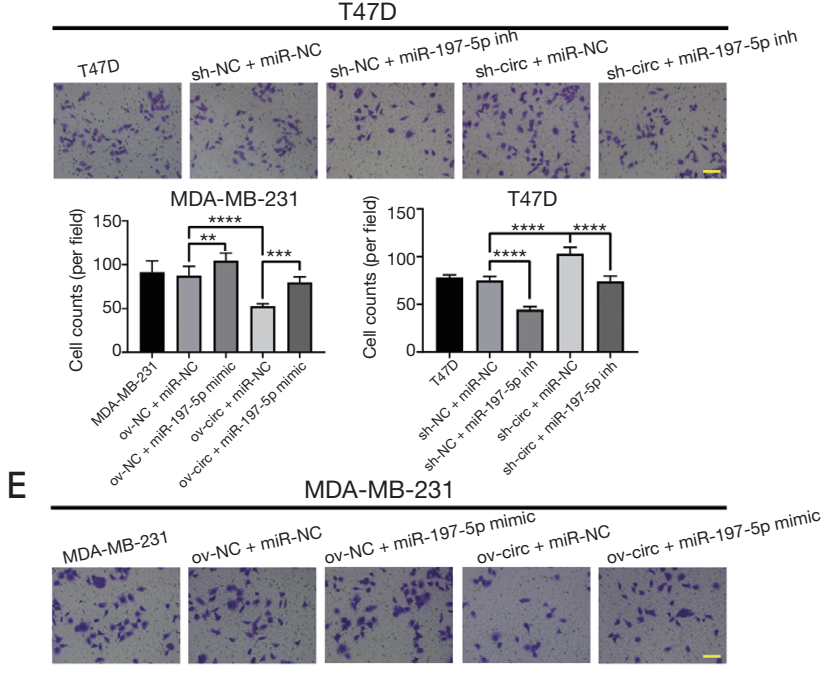

T47D
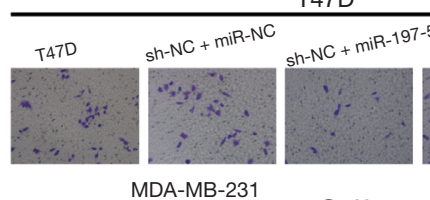

97-5p inh $+m i R-N$
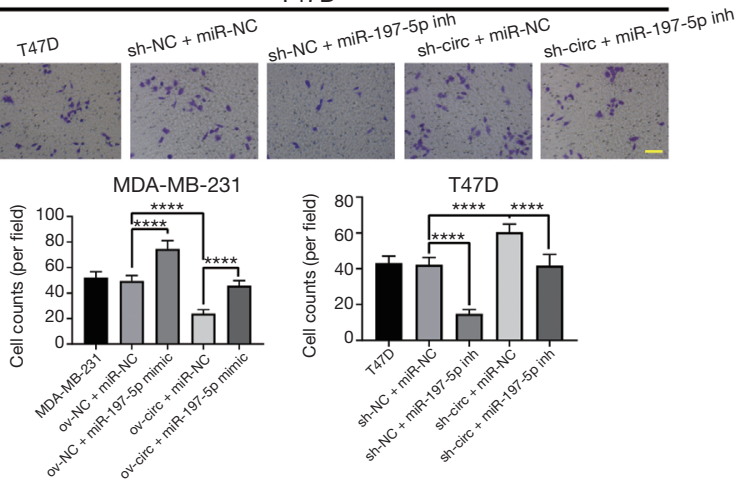

Figure 5 hsa_circ_0006220 inhibited TNBC cell growth, migration, and invasion by sponging miR-197-5p. (A) After transfection with the hsa_circ_0006220 expression vector (ov-circ) or the mock (ov-NC) and the miR-197-5p mimic or the miR-NC in MDA-MB-231 cells, as well as transfection with the hsa_circ_0006220 siRNA (sh-circ) or the negative control and the miR-197-5p inhibitor (miR-197-5p inh) or the miR-NC in T47D cells, relative expressions of hsa_circ_0006220 were examined in each group by RT-PCR. (B) The growth curves of cells transfected with the specified vectors were constructed by MTT assays. (C) Cell migration capacities were detected by wound-healing assays after being transfected with the specified vectors in cells. Scale bar, $200 \mu \mathrm{m}$. (D,E) Cell migration and invasion abilities were analyzed by Transwell assays after being transfected with the specified vectors in cells. Stain method: $0.5 \%$ crystal violet dyes. Scale bar, $100 \mu \mathrm{m}$. Data are presented as mean \pm standard deviation $(\mathrm{n}=3)$. ${ }^{* *}, \mathrm{P}<0.01 ;{ }^{* * *}, \mathrm{P}<0.001 ;{ }^{* * *}, \mathrm{P}<0.0001$. 
A

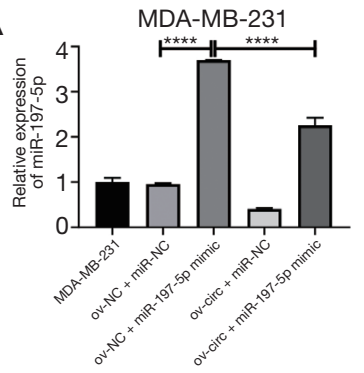

B

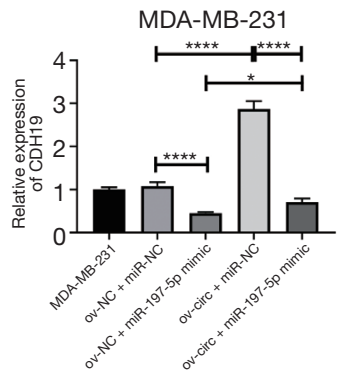

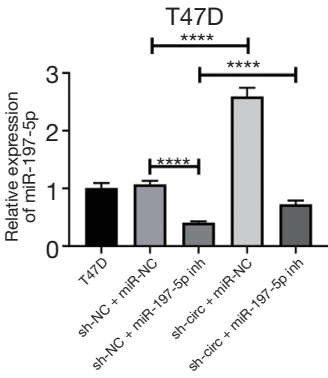

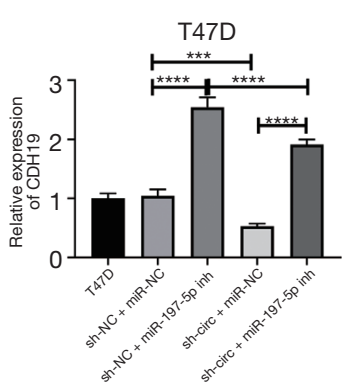

C
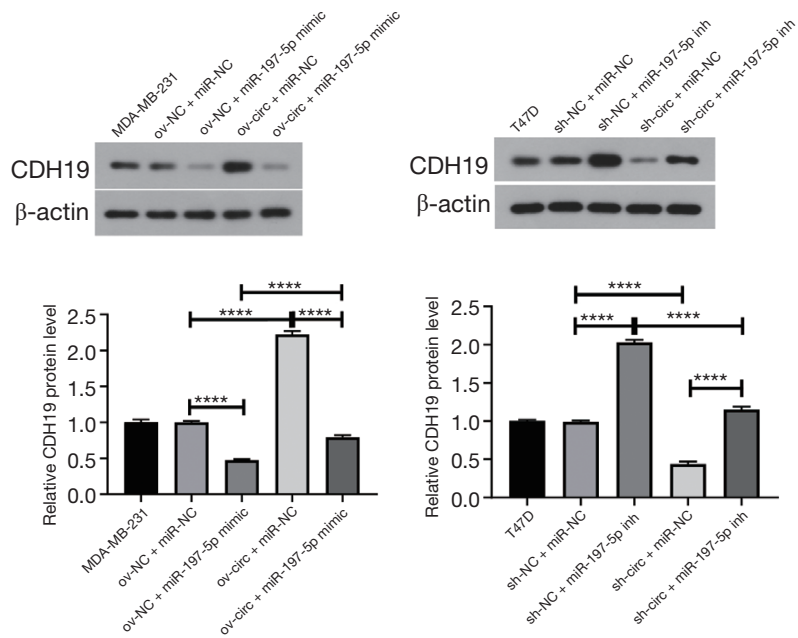

Figure 6 hsa_circ_0006220 regulates CDH19 expression by sponging miR-197-5p. (A) After transfection with the hsa_circ_0006220 expression vector (ov-circ) or the mock (ov-NC) and the miR-197-5p mimic or the miR-NC in MDA-MB-231 cells, as well as transfection with the hsa_circ_0006220 siRNA (sh-circ) or the negative control and the miR-197-5p inhibitor (miR-197-5p inh) or the miR-NC in T47D cells, the levels of miR-197-5p were tested by RT-PCR in each group. (B) Relative mRNA levels of CDH19 were examined in each group by RT-PCR, and (C) Relative protein levels of CDH19 were detected in cells by western blot after being transfected with the specified vectors. Data are presented as mean \pm standard deviation $(\mathrm{n}=3)$. ${ }^{*}, \mathrm{P}<0.05 ;{ }^{* * *}, \mathrm{P}<0.001 ;{ }^{* * *}, \mathrm{P}<0.0001$.

\section{Discussion}

TNBC is a subtype of $B C$, which is characterized by the absence of ER, PR and HER-2. This type of BC has a poor prognosis, a high recurrence rate, is prone to early metastasis, and lacks an effective targeted therapy (2,20-22). Thus, exploring a new therapeutic target could improve the survival of TNBC patients. CircRNAs are a special type of stable and conserved endogenous RNAs that are now a hotspot of RNA research $(4,5,23)$. However, studies about circRNAs in relation to TNBC remain limited. Discovering and understanding the biological roles of a new circRNAs in TNBC could be beneficial in the treatment of TNBC.

In our previous study, a novel circRNA (i.e., hsa circ_0006220) was found to be obviously downregulated in BC tissues. The expression of hsa_circ_0006220 also correlated with histological type and lymph node metastasis. All these results suggested a correlation between hsa circ_0006220 and the malignant degrees of BC. In the present study, we found that hsa_circ_0006220 was not only downregulated in human $\mathrm{BC}$ tissues and cells but also in TNBC tissues and cells relative to other subtypes of BC tissues and cells. These findings led us to further investigate the biological roles of hsa_circ_0006220 in TNBC. We selected a TNBC cell line (i.e., MDA-MB-231), which has the lowest expression of hsa_circ_0006220, and a non-TNBC cell line (i.e., T47D), which has the highest expression of hsa_circ_0006220, for our subsequent studies. We found that the ectopic expression of hsa circ_0006220 strikingly inhibited TNBC cell proliferation and the cells' metastatic properties. Conversely, the silencing of hsa_circ_0006220 remarkably promoted cell growth and metastatic properties in T47D cells. Thus, the tumor suppressor roles of hsa_circ_0006220 on TNBC progression were identified in vitro.

Numerous of circRNAs have been reported to function as miRNA sponges in cancers $(24,25)$, and circRNAs play roles in the development of tumors by their diseaseassociated target miRNAs (26). Previously, we identified 5 possible target miRNAs of hsa_circ_0006220 (14). Among them, miR-197-5p was found to be regulated by hsa circ_0006220. miR-197 was reported to be a biomarker of different cancers (27). The expression of miR-197 was also notably increased in the serum of patients with $\mathrm{BC}(15)$ and was elevated in male BC tissues (28). Thus, we 
presumed that the biological roles of hsa_circ_0006220 in TNBC might be mediated by miR-197-5p. In the present study we found that hsa_circ_0006220 inhibited miR-197$5 \mathrm{p}$ expression. The dual-luciferase reporter assay further suggested that hsa_circ_0006220 directly sponges miR-197$5 \mathrm{p}$. The Pearson correlation analysis revealed a negative correlation between miR-197-5p and hsa_circ_0006220 in $\mathrm{BC}$ tissues. Thus, the interaction between miR-197-5p and hsa_circ_0006220 was identified.

Further, CDH19 was predicted to be a downstream gene of miR-197-5p. CDH19 is regarded as a member of cadherin and establishes and maintains intercellular connections (17-19,29,30). In addition, CDH19 was also found to be downregulated in TNBC cells and tissues, and its level was positively correlated with hsa_circ_0006220. Further, we found that the overexpression of hsa_circ_0006220 significantly boosted CDH19 expression in TNBC cells. The rescue assays showed that enhancing miR-197-5p partially reduced the tumor suppressing functions of overexpressing hsa_circ_0006220 in TNBC cells, while inhibiting miR197-5p had the opposite effect in T47D cells. These results showed that the hsa_circ_0006220/miR-197-5p/CDH19 pathway might help in the progression of TNBC. However, large-scale studies need to be conducted to validate the diagnostic and therapeutic performance of hsa_circ_0006220.

Notwithstanding its limitations, this study found evidence that hsa_circ_0006220 affects the novel cadherin gene CDH19, and thus affects the progression of TNBC cells. However, research on CDH19 is still scarce. The question of how CDH19 affects the progression of TNBC requires further exploration. Additionally, the number of patient samples in this paper are few, it needs to be expanded to verify the relationship between TNBC and hsa circ_0006220. Multicenter-trials also need to be conducted to elucidate the roles of hsa_circ_0006220 in the future.

Collectively, these data showed that hsa_circ_0006220 is downregulated in TNBC tissues and cells and its downstream mechanisms, which inhibits the progression of TNBC cells. hsa_circ_0006220 might serve as a potential diagnostic marker and therapeutic target for TNBC patients. The hsa_circ_0006220/miR-197-5p/ CDH19 pathway provides insights into the mechanism of progression of TNBC.

\section{Acknowledgments}

We would like to thank The First Affiliated Hospital of China Medical University for its technical support.
Funding: The project is supported by the National Youth Fund of National Natural Science Foundation (81702622).

\section{Footnote}

Reporting Checklist: The authors have completed the MDAR checklist. Available at https://dx.doi.org/10.21037/ atm-21-2934

Data Sharing Statement: Available at https://dx.doi. org/10.21037/atm-21-2934

Conflicts of Interest: All authors have completed the ICMJE uniform disclosure form (available at https://dx.doi. org/10.21037/atm-21-2934). All authors report that the project is supported by the National Youth Fund of National Natural Science Foundation (81702622). The authors have no other conflicts of interest to declare.

Ethical Statement: The authors are accountable for all aspects of this work, including ensuring that any questions related to the accuracy or integrity of any part of the work have been appropriately investigated and resolved. The study was approved by the Ethics Committee of The First Affiliated Hospital of China Medical University (No. AF0G-03-1.0-02). All procedures performed in this study involving human participants were conducted in accordance with the Declaration of Helsinki (as revised in 2013). Informed consent was obtained from all of the patients.

Open Access Statement: This is an Open Access article distributed in accordance with the Creative Commons Attribution-NonCommercial-NoDerivs 4.0 International License (CC BY-NC-ND 4.0), which permits the noncommercial replication and distribution of the article with the strict proviso that no changes or edits are made and the original work is properly cited (including links to both the formal publication through the relevant DOI and the license). See: https://creativecommons.org/licenses/by-nc-nd/4.0/.

\section{References}

1. DeSantis CE, Ma J, Goding Sauer A, et al. Breast cancer statistics, 2017, racial disparity in mortality by state. CA Cancer J Clin 2017;67:439-48.

2. Foulkes WD, Smith IE, Reis-Filho JS. Triple-negative breast cancer. N Engl J Med 2010;363:1938-48.

3. Zhao S, Zuo WJ, Shao ZM, et al. Molecular subtypes and 
precision treatment of triple-negative breast cancer. Ann Transl Med 2020;8:499.

4. Memczak S, Jens M, Elefsinioti A, et al. Circular RNAs are a large class of animal RNAs with regulatory potency. Nature 2013;495:333-8.

5. Lasda E, Parker R. Circular RNAs: diversity of form and function. RNA 2014;20:1829-42.

6. Wang H, Xiang Y, Hu R, et al. Research progress of circular RNA in digestive tract tumors: a narrative review. Transl Cancer Res 2020;9:7632-41.

7. Han B, Chao J, Yao H. Circular RNA and its mechanisms in disease: From the bench to the clinic. Pharmacol Ther 2018;187:31-44.

8. Chen Y, Yang F, Fang E, et al. Circular RNA circAGO2 drives cancer progression through facilitating HuRrepressed functions of AGO2-miRNA complexes. Cell Death Differ 2019;26:1346-64.

9. Hansen TB, Jensen TI, Clausen BH, et al. Natural RNA circles function as efficient microRNA sponges. Nature 2013;495:384-8.

10. Li XN, Wang ZJ, Ye CX, et al. RNA sequencing reveals the expression profiles of circRNA and indicates that circDDX17 acts as a tumor suppressor in colorectal cancer. J Exp Clin Cancer Res 2018;37:325.

11. Liu G, Shi H, Deng L, et al. Circular RNA circ-FOXM1 facilitates cell progression as ceRNA to target PPDPF and MACC1 by sponging miR-1304-5p in non-small cell lung cancer. Biochem Biophys Res Commun 2019;513:207-12.

12. Tang H, Huang X, Wang J, et al. circKIF4A acts as a prognostic factor and mediator to regulate the progression of triple-negative breast cancer. Mol Cancer 2019;18:23.

13. Chen B, Wei W, Huang X, et al. circEPSTI1 as a Prognostic Marker and Mediator of Triple-Negative Breast Cancer Progression. Theranostics 2018;8:4003-15.

14. Liu C, Chen M, Shi Y. Downregulation of hsa_ circ_0006220 and its correlation with clinicopathological factors in human breast cancer. Gland Surg 2021;10:816-25.

15. Shaker O, Maher M, Nassar Y, et al. Role of microRNAs -29b-2, -155, -197 and -205 as diagnostic biomarkers in serum of breast cancer females. Gene 2015;560:77-82.

16. Tang T, Cheng Y, She Q, et al. Long non-coding RNA TUG1 sponges miR-197 to enhance cisplatin sensitivity in triple negative breast cancer. Biomed Pharmacother 2018;107:338-46.

17. Kools P, Van Imschoot G, van Roy F. Characterization of three novel human cadherin genes (CDH7, CDH19, and $\mathrm{CDH} 20$ ) clustered on chromosome 18q22-q23 and with high homology to chicken cadherin-7. Genomics 2000;68:283-95

18. Kremmidiotis G, Baker E, Crawford J, et al. Localization of human cadherin genes to chromosome regions exhibiting cancer-related loss of heterozygosity. Genomics 1998;49:467-71.

19. Tervasmäki A, Winqvist R, Jukkola-Vuorinen A, et al. Recurrent CYP2C19 deletion allele is associated with triple-negative breast cancer. BMC Cancer 2014;14:902.

20. Guestini F, McNamara KM, Ishida T, et al. Triple negative breast cancer chemosensitivity and chemoresistance: current advances in biomarkers indentification. Expert Opin Ther Targets 2016;20:705-20.

21. Ali AM, Ansari JAK, El-Aziz NMA, et al. Triple Negative Breast Cancer: A Tale of Two Decades. Anticancer Agents Med Chem 2017;17:491-9.

22. de Paula BHR, Kumar S, Morosini FM, et al. Realworld assessment of the effect of impact of tumor size on pathological complete response rates in triple negative breast cancer after neoadjuvant chemotherapy. Chin Clin Oncol 2020;9:78.

23. Petkovic $\mathrm{S}$, Muller S. RNA circularization strategies in vivo and in vitro. Nucleic Acids Res 2015;43:2454-65.

24. Salzman J, Gawad C, Wang PL, et al. Circular RNAs are the predominant transcript isoform from hundreds of human genes in diverse cell types. PLoS One 2012;7:e30733.

25. Xu H, Guo S, Li W, et al. The circular RNA Cdr1as, via miR-7 and its targets, regulates insulin transcription and secretion in islet cells. Sci Rep 2015;5:12453.

26. Ghosal S, Das S, Sen R, et al. Circ2Traits: a comprehensive database for circular RNA potentially associated with disease and traits. Front Genet 2013;4:283.

27. Wang DD, Chen X, Yu DD, et al. miR-197: A novel biomarker for cancers. Gene 2016;591:313-9.

28. Lehmann U, Streichert T, Otto B, et al. Identification of differentially expressed microRNAs in human male breast cancer. BMC Cancer 2010;10:109.

29. Aberle H, Schwartz H, Kemler R. Cadherin-catenin complex: protein interactions and their implications for cadherin function. J Cell Biochem 1996;61:514-23.

30. Mareel M, Berx G, Van Roy F, et al. Cadherin/catenin complex: a target for antiinvasive therapy? J Cell Biochem 1996;61:524-30.

(English Language Editor: L. Huleatt)

Cite this article as: Shi Y, Han T, Liu C. CircRNA hsa circ_0006220 acts as a tumor suppressor gene by regulating miR-197-5p/CDH19 in triple-negative breast cancer. Ann Transl Med 2021;9(15):1236. doi: 10.21037/atm-21-2934 
Supplementary

Table S1 The sequences of the primers in this study

\begin{tabular}{lll}
\hline RNA & Forward (5'-3') & Reverse (5'-3') \\
\hline hsa_circ_0006220 & CCCTGCTGAACCTGAAAC & CACTCCTCCTTGGTCTTG \\
CDH19 & CATTTGTAGGCGTGGTGT & GTGGCTGTAATACTTAGGTTGT \\
TADA2A & ATGGACTGTGGCTTGGA & TTCAGGTTCAGCAGGGTA \\
B-actin & CGGGAATCGTGCGTGAC & GTCAGGCAGCTCGTAGCTCTT \\
miR-197-5p & CGGGTAGAGAGGGCAGTGGGAGG & GCAGGGTCCGAGGTATTC \\
U6 & GCTTCGGCAGCACATATACT & GTGCAGGGTCCGAGGTATTC \\
\hline
\end{tabular}

Table S2 The sequences of the siRNAs

\begin{tabular}{ll}
\hline RNA & Sequence (5'-3') \\
\hline hsa-circ-0006220 siRNA & ACAUCCUGCAGUGAAAUGGTT \\
si-NC & ACGUGACACGUUCGGAGATT \\
\hline
\end{tabular}

Table S3 miR-197-5p inhibitor sequence

\begin{tabular}{ll}
\hline RNA & Sequence $\left(5^{\prime}-3^{\prime}\right)$ \\
\hline miR-197-5p inhibitor & CCUCCCACUGCCCUCUCUACCCG \\
Inhibitor NC & UUGUACUACACAAAAGUACUG \\
\hline
\end{tabular}

